\title{
Small matches and charitable giving: Evidence from a natural field experiment ${ }^{\hbar}$
}

\author{
Dean Karlan ${ }^{\text {a,b,f }}$, John A. List ${ }^{\text {b,c,d,*, Eldar Shafir }}{ }^{\text {b,e }}$ \\ a Yale University, Department of Economics, 27 Hillhouse Avenue, New Haven CT 06511, United States \\ b Innovations for Poverty Action \\ c The University of Chicago, Department of Economics, 1126 East 59th Street, Chicago, IL 60637, United States \\ d NBER \\ e Princeton University, Department of Psychology and Woodrow Wilson School, Princeton, NJ 08540, United States \\ ${ }^{\mathrm{f}}$ Massachusetts Institute of Technology, Jameel Poverty Action Lab
}

\section{A R T I C L E I N F O}

\section{Article history:}

Received 7 October 2009

Received in revised form 15 November 2010

Accepted 19 November 2010

Available online 28 November 2010

\section{Keywords:}

Charitable giving

Matching

Warm and cold list donors

\begin{abstract}
A B S T R A C T
To further our understanding of the economics of charity, we conducted a natural field experiment. Making use of two direct mail solicitations sent to nearly 20,000 prior donors to a charity, we tested the effectiveness of $\$ 1: \$ 1$ and $\$ 1: \$ 3$ matching grants on charitable giving. We find only weak evidence that either of the matches work; in fact, for the full sample, the match only increased giving after the match deadline expired. Yet, the aggregation masks important heterogeneities: those donors who are actively supporting the organization tend to be positively influenced whereas lapsed givers are either not affected or adversely affected. Furthermore, some presentations of the match can do harm, e.g., when an example amount given is high ( $\$ 75)$ and the match ratio is below $\$ 1: \$ 1$. Overall, the results help clarify what might cause people to give and provide further evidence that larger match ratios are not necessarily superior to smaller match ratios.
\end{abstract}

(c) 2010 Elsevier B.V. All rights reserved.
The charitable marketplace represents an interesting set of three major agents (Andreoni, 2007). First, are the donors, who provide the resources for private organizations to produce public goods. A rich theoretical and empirical literature has developed in the last few decades that explores what motivates individual giving (see Andreoni, 2006, for an excellent overview). Second, is the government, which affects decisions on the margin through its use of the tax code, and the level of transfers it sends to charitable operations. ${ }^{1}$ The final set of actors is charitable organizations, which develop strategies intended to attract resources to produce public goods. In the U.S., this final set of actors has been reasonably successful, as charitable monetary gifts have been 2\% or more of GDP since 1998 .

Even though the stakes in understanding the charitable market linkages are clearly quite high, until recently economists have largely ignored the link between individuals and charitable fundraisers. A set of recent natural field experiments has begun to lend insights to this area (see the overview in List, forthcoming). One early natural field experiment

\footnotetext{
th Thanks to the seminar participants at the Middlebury Conference on Philanthropy, 2009.

* Corresponding author. The University of Chicago, Department of Economics, 1126 East 59th Street, Chicago, IL 60637, United States.

E-mail addresses: dean.karlan@yale.edu (D. Karlan), jlist@uchicago.edu (J.A. List), shafir@princeton.edu (E. Shafir).

${ }^{1}$ Feldstein (1975), Clotfelter (1985), Randolph (1995), and Auten et al. (2002) are examples of empirical work that examines price effects via rebate mechanisms (such as changes in tax deductions) through which charitable contributions can be used to reduce one's tax burden. Andreoni and Payne (forthcoming) is a recent empirical contribution that explores how fundraisers respond to government transfers.
}

is List and Lucking-Reiley (2002). Exploring how seed money and refunds influenced giving, they solicited contributions from 3000 Central Floridian residents, randomly assigned to treatment and control. They found that increased seed money sharply increased both the participation rate and the average gift size received from participating donors. In addition, they found that refunds only had a small effect.

Subsequent studies have lent similar insights on the importance of seed money. For example, Rondeau and List (2008) made use of a natural field experiment, dividing 3000 direct mail solicitations to Sierra Club supporters into four treatments asked to support the expansion of a K-12 environmental education program. They found that the announcement of seed money increased the participation rate of potential donors by $23 \%$ and total dollar contributions by $18 \%$, compared to an identical campaign in which no announcement of leadership gift was made. Related to this line of work, Frey and Meier (2004) provide empirical evidence from a clever natural field experiment that suggests individual comparisons are important when making the donation decision. Using a series of innovative natural field experiments with National Public Radio, Croson and Shang $(2005,2008)$ find similar results, whether they focus on estimating the effect of upward or downward social information.

Karlan and List (2007) expand this area of research by exploring the comparative static effects of varying prices directly. They solicited contributions from more than 50,000 supporters of a liberal organization by randomizing them into several groups to explore whether upfront monies used as matching funds promote giving. They found that simply announcing that a match is available considerably increases the revenue per solicitation - by $19 \%$. In addition, the match offer significantly increased the probability that 
an individual donates - by $22 \%$. Yet, while the match treatments relative to a control group increased the probability of donating, they found that larger match ratios - \$3:\$1 (i.e., \$3 match for every $\$ 1$ donated) and $\$ 2: \$ 1$ - relative to smaller match ratios ( $\$ 1: \$ 1)$ have no additional impact.

Unfortunately, Karlan and List (2007) did not explore match rates less than $\$ 1: \$ 1$, excluding what may have proven to be potentially lucrative match rates for the charity. In what follows, beyond exploring prices over a different range than Karlan and List (2007) from a baseline of no match to $\$ 1: \$ 3$ to $\$ 1: \$ 1$ - we also examine whether cold and warm list donors respond differently to match solicitations. In doing so, we maintain congruity with Karlan and List (2007) by also exploring giving patterns to a liberal (but different) organization that also focuses on civil justice issues. Although the organization is different, it is of similar spirit to the organization used in Karlan and List (2007). This is important in light of the estimates presented in Feldstein (1975), which suggest that price elasticities vary by type of charitable organization. ${ }^{2}$

We find some interesting data patterns. Consistent with Karlan and List (2007), we find that in the full sample giving patterns are not different across the various match rates, either during the experiment or afterwards. At odds with Karlan and List (2007), however, we find that in the aggregate data the match has little effect within the fundraising drive. ${ }^{3}$ Yet, the aggregation masks important heterogeneity, which was not present in the earlier Karlan and List study. For instance, there is some evidence that warm list donors respond more favorably to match funds than cold list agents, and there is weak evidence that the match influenced giving months after the experiment. This result is consistent with the short versus long term effects found in McManus and Bennet (forthcoming) in this special issue. Furthermore, we find that when the example matching amount is $\$ 25$ instead of $\$ 1$ (i.e., "For example, if you give $\$ 75$, the matching donor will give $\$ 25$ " versus "For example, if you give $\$ 3$, the matching donor will give \$1."), then the ratio of the match does matter, in particular for those who have not given before. In this case, the larger example amount actually causes harm, whereas the aggregate effect is nil.

The remainder of this paper proceeds as follows. The next section details the experimental design. Section 2 discusses the results. Section 3 concludes.

\section{Experimental design}

In a first round of experimentation with matching ratios (Karlan and List, 2007), we worked with a liberal organization that focuses on social and policy issues related to civil liberties. The Organization that we worked with in this round was a different organization that also focuses on civil justice issues. Anecdotal reports from the Organization suggest that their typical supporter is liberal politically, but not as intensely as the Organization involved in Karlan and List (2007), where $89 \%$ of supporters self-reported voting for Gore in the 2000 Presidential Election. No similar data are available for the Organiza-

\footnotetext{
${ }^{2}$ Note that throughout this paper elasticity refers to the donor decision, not the perspective of the organization. In other words, we do not include the match in the numerator when calculating percent change in amount raised as price changes. This is consistent with other research in understanding donor behavior (Karlan and List, 2007). In general, when one considers how such results might influence policy, we believe that framing is of great import (see also Levitt and List, 2007). For example, what we learn from the lab experiments of Eckel and Grossman (2003) is that matches work much better than rebates. This suggests that these two situations are treated differently in the donor's mind, despite the economists' ability to make them equal on economic/analytical terms. This has important implications for how we interpret elasticities, and also for policy on framing, if one wants to use the tax code to change behavior and use experimental data to inform the policy decision it implies that we need to use similarly-framed situations.

${ }^{3}$ We define the fundraising drive as the period between the delivery of the first mailer (allowing a few days for receipt of the mailer) and the final match deadline.
}

tion in the present experiment, but the organization believes that their core issue crosses over political lines more so than the issue on which the 2007 organization focuses. The Organization is a charity under United States Internal Revenue Service code 501(c)3, hence donations are tax-deductible for federal income taxes. This Organization typically solicits donations roughly four times per year and our field experiment was conducted through two of those fundraising drives.

Our sample consists of 19,959 individuals who have given to the Organization at least once since $1983 .{ }^{4}$ If one uses the standard definitions in the industry - i.e., a warm list person is a donor who has given in the past year ( 2 years; 5 years) - we have a span of individual giving that permits an analysis of whether warm list agents respond differently to match opportunities than cold list agents. Of course, care must be taken when interpreting results associated with this classification because donor type is not experimentally manipulated; nevertheless, the exercise is instructive in that it provides insights into some of the factors that might influence giving among different donor types, and the most effective ways to address those factors.

All individuals received a one page letter identical in all respects, except (1) treatment letters included a modified postscript paragraph in which it was announced that a donor will match their donation, along with details of the match, and (2) treatment letter reply cards repeated the details of the match. The remainder of the letter followed the Organization's usual fundraising practices. The letter briefly covered the contents of an accompanying newsletter.

For the fundraising drive, 19,959 prior donors were assigned to one of four groups, forming our $2 \times 2$ full factorial design:

1. Matching treatment + urgency treatment (6580): Received a matching offer, and an urgency statement. The urgency statement was a sentence, printed in all capital, bolded letters, intended to communicate the need for immediate action. This statement differed slightly across the September and December mailers. For the September mailer, the urgency statement began the reply card paragraph and the post script paragraph with the sentence "NOW IS THE TIME TO GIVE!" For the December mailer, the urgency statement "NOW IS THE TIME TO GIVE!" was placed at the beginning of the reply card paragraph, while a different urgency statement, "NOW IS THE TIME TO JOIN THE FIGHT!" appeared at the end of the front page paragraph.

2. Matching treatment + urgency control (6572): Received a matching offer, but no statement of urgency. In lieu of the urgency statement, the September reply card paragraph began with "I Want to Keep Fighting for Equality and Justice" and the postscript paragraph merely omitted the urgency statement. The December reply card paragraph began with "Let's Help Our Children Learn to Live Together as Brothers," and the front page paragraph omitted the urgency statement.

3. Matching control + urgency treatment (3424): No matching grant offered, but contained the urgency statement "Now is the Time to Give."

4. Matching control + urgency control (3383): Neither the matching grant offered nor the urgency statement.

For those agents receiving the matching grant treatment, we further randomized along three dimensions:

1. Ratio of matching grant: The matching offer was either a $\$ 1: \$ 1$ offer (6438) or a $\$ 1: \$ 3$ offer (6714). A $\$ 1: \$ 1$ ratio means that for every dollar the individual donates to the Organization, a lead donor will also donate $\$ 1$; hence the charity receives $\$ 2$. The $\$ 1: \$ 3$

\footnotetext{
439 individuals did not have any prior giving history, but were kept in the sample.
} 
ratio means that for every dollar the individual donates to the Organization, a lead donor will also donate $\$ 0.33$; hence the charity receives $\$ 1.33$.

2. Deadline of matching grant: During the September fundraising drive, individuals offered the match treatment were given one of two donation deadlines: October 31st (6385) or December 31st (6204). All individuals in the December fundraising drive were given the December 31st deadline (including individuals given the October 31st deadline in the September mailer, who were subsequently told that the match had been extended until December 31st).

3. Amount of matching grant: During the September fundraising drive, amounts matched as a result of grant were framed as either $\$ 1$ for each $\$ 3$ donated (6287), or $\$ 25$ for each $\$ 75$ donated (6302).

Of the total sample size of $19,959,18,883$ received a mailer both in September and in December, and 1126 received only the December mailer. Whether one or both mailers were received was not randomized. All results specifications include a control variable for receiving only the December mailer.

The December mailer differed from the September mailer in two respects: (1) the December mailer was a two-page letter, unaccompanied by a newsletter, which discussed a pressing legal issue that the Organization was fighting, and (2) the match offer, if present, was mentioned in a modified paragraph on the front page of the letter and in the postscript. All individuals offered the matching treatment were given the same deadline (December 31st) and received the same match ratio as they did in the September mailer. All individuals, regardless of matching treatment assignment, received the same urgency statement as they did in the September mailer.

\section{Experimental results}

Table 1 provides a summary of the households randomly placed into each treatment. An important consideration is that balance was not achieved across all of the important treatment cells. For example, considering an F-test of joint significance, while we find that variables such as size of most recent donation and average size of previous donations are balanced across treatment, other variables such as median household income (from zip-code level census data) and whether the donor had already given in 2006 were not balanced. We therefore include controls for all variables observable at baseline in the empirical analysis presented below.

Table 2 presents the core results for two periods: (1) the experiment period of September 6, 2006 to December 31, 2006 (i.e. the pre-deadline period) and (2) the post-experiment period of January 1, 2007 to June 24, 2007. Our specifications cover three outcome measures: (1) a continuous variable for total amount given in each period, (2) the logarithm of ( $1+$ the amount given in each period), and (3) a binary variable equal to one if any donation is made in each period. We estimate four OLS specifications for each period and outcome measure:

$$
\begin{aligned}
& \text { (1) } Y_{i}=\alpha_{0}+\alpha_{1} M 1_{i}+\alpha_{2} M 3_{i}+\alpha_{3} S_{i}+\alpha_{4} U_{i}+\alpha_{5} R_{i}+\alpha_{6} D_{i}+\varepsilon_{i} \\
& \text { (2) } Y_{i}=\beta_{0}+\beta_{1} M_{i}+\beta_{2} S_{i}+\beta_{3} U_{i}+\beta_{4} R_{i}+\beta_{5} D_{i}+\varepsilon_{i} \\
& \text { (3) } Y_{i}=\gamma_{0}+\gamma_{1} M_{i}+\gamma_{2} S_{i}+\gamma_{3} U_{i}+\gamma_{4} R_{i}+\gamma_{5} M_{i} U_{i}+\gamma_{6} D_{i}+\varepsilon_{i} \\
& \text { (4) } \mathrm{Y}_{\mathrm{i}}=\lambda_{0}+\lambda_{1} \mathrm{M}_{\mathrm{i}}+\lambda_{2} \mathrm{M}_{3}+\lambda_{3} \mathrm{~S}_{\mathrm{i}}+\lambda_{4} \mathrm{U}_{\mathrm{i}}+\lambda_{5} \mathrm{R}_{\mathrm{i}}+\lambda_{6} \mathrm{M}_{\mathrm{i}} \mathrm{R}_{\mathrm{i}} \\
& +\lambda_{7} \mathrm{M} 3_{\mathrm{i}} \mathrm{R}_{\mathrm{i}}+\lambda_{8} \mathrm{D}_{\mathrm{i}}+\varepsilon_{\mathrm{i}}
\end{aligned}
$$

\begin{tabular}{|c|c|c|c|c|c|}
\hline & Control & Match $(1: 1)$ & Match (1:3) & Urgency & $\begin{array}{l}P \text {-value from F-test of joint } \\
\text { significance of }(2),(3), \\
\text { and }(4), \text { relative to }(1)\end{array}$ \\
\hline Baseline & $(1)$ & $(2)$ & $(3)$ & $(4)$ & $(5)$ \\
\hline Size of most recent donation, prior to experiment (in dollars) & $\begin{array}{l}75.031 \\
(2.570)\end{array}$ & $\begin{array}{l}66.929 \\
(2.505) \\
{[0.024]}\end{array}$ & $\begin{array}{l}71.881 \\
(2.611) \\
{[0.390]}\end{array}$ & $\begin{array}{l}71.803 \\
(2.027) \\
{[0.763]}\end{array}$ & 0.162 \\
\hline $\begin{array}{l}\text { Days between September 6, } 2006 \text { and most recent donation } \\
\text { prior to the September mailer }\end{array}$ & $\begin{array}{l}382.703 \\
(3.899)\end{array}$ & $\begin{array}{l}407.683 \\
(3.594) \\
{[0.000]}\end{array}$ & $\begin{array}{l}375.060 \\
(3.569) \\
{[0.191]}\end{array}$ & $\begin{array}{l}387.882 \\
(2.971) \\
{[0.788]}\end{array}$ & 0.000 \\
\hline $\begin{array}{l}\text { Donor gave in year prior to the match, prior to experiment } \\
\text { (proportion) }\end{array}$ & $\begin{array}{l}0.445 \\
(0.006)\end{array}$ & $\begin{array}{l}0.411 \\
(0.006) \\
{[0.000]}\end{array}$ & $\begin{array}{l}0.455 \\
(0.006) \\
{[0.350]}\end{array}$ & $\begin{array}{l}0.435 \\
(0.005) \\
{[0.737]}\end{array}$ & 0.000 \\
\hline $\begin{array}{l}\text { Total sum of donations ever given, prior to experiment } \\
\text { (in } \$ 1000 \text { s) }\end{array}$ & $\begin{array}{l}0.635 \\
(0.029)\end{array}$ & $\begin{array}{l}0.433 \\
(0.022) \\
{[0.000]}\end{array}$ & $\begin{array}{l}0.553 \\
(0.028) \\
{[0.0382]}\end{array}$ & $\begin{array}{l}0.560 \\
(0.024) \\
{[0.253]}\end{array}$ & 0.000 \\
\hline $\begin{array}{l}\text { Average size of donation ever given, prior to experiment } \\
\text { (in } \$ 1000 \text { s) }\end{array}$ & $\begin{array}{l}0.063 \\
(0.002)\end{array}$ & $\begin{array}{l}0.058 \\
(0.002) \\
{[0.037]}\end{array}$ & $\begin{array}{l}0.063 \\
(0.002) \\
{[0.845]}\end{array}$ & $\begin{array}{l}0.062 \\
(0.001) \\
{[0.928]}\end{array}$ & 0.146 \\
\hline $\begin{array}{l}\text { Total sum of donations given over past } 3 \text { years, prior to } \\
\text { experiment (in } \$ 1000 \text { s) }\end{array}$ & $\begin{array}{l}0.170 \\
(0.006)\end{array}$ & $\begin{array}{l}0.143 \\
(0.005) \\
{[0.001]}\end{array}$ & $\begin{array}{l}0.155 \\
(0.006) \\
{[0.089]}\end{array}$ & $\begin{array}{l}0.160 \\
(0.005) \\
{[0.205]}\end{array}$ & 0.006 \\
\hline Median household income, by zip-code (in $\$ 1000$ s) & $\begin{array}{l}53.437 \\
(0.303)\end{array}$ & $\begin{array}{l}51.450 \\
(0.290) \\
{[0.000]}\end{array}$ & $\begin{array}{l}51.361 \\
(0.289) \\
{[0.000]}\end{array}$ & $\begin{array}{l}52.248 \\
(0.239) \\
{[0.150]}\end{array}$ & 0.000 \\
\hline $\begin{array}{l}\text { Proportion of income donated (sum of donations over past } \\
3 \text { years/average HH income over } 3 \text { years, by zip-code) }\end{array}$ & $\begin{array}{l}0.003 \\
(0.000)\end{array}$ & $\begin{array}{l}0.003 \\
(0.000) \\
{[0.414]}\end{array}$ & $\begin{array}{l}0.003 \\
(0.000) \\
{[0.085]}\end{array}$ & $\begin{array}{l}0.003 \\
(0.000) \\
{[0.083]}\end{array}$ & 0.283 \\
\hline \multicolumn{6}{|l|}{ Sub-Treatments } \\
\hline $\begin{array}{l}\text { Received the September mailer with a short deadline for } \\
\text { match offer (October 31) }\end{array}$ & & $\begin{array}{l}0.463 \\
(0.006)\end{array}$ & $\begin{array}{l}0.507 \\
(0.006) \\
{[0.000]}\end{array}$ & $\begin{array}{l}0.324 \\
(0.005) \\
{[0.084]}\end{array}$ & 0.000 \\
\hline September mailer framed match as $\$ 25$ for every $\$ 25$ or $\$ 75$ & & $\begin{array}{l}0.474 \\
(0.006)\end{array}$ & $\begin{array}{l}0.484 \\
(0.006) \\
{[0.0464]}\end{array}$ & $\begin{array}{l}0.323 \\
(0.005) \\
{[0.191]}\end{array}$ & 0.013 \\
\hline Number of Observations & 6807 & 6438 & 6714 & 10,004 & \\
\hline
\end{tabular}

where $Y_{i}$ is equal to one if individual $i$ donated in the period, or equal to the total amount (or $\log [1+$ total amount $]$ ) donated by individual $i$

Table 1

Orthogonality Checks.

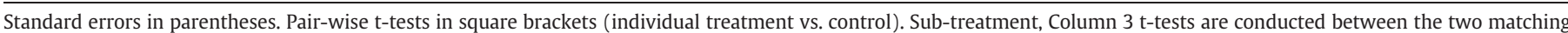
treatments. 
Table 2

Core, Total Sum of Donation \& Whether or Not Donation Was Given.

\begin{tabular}{|c|c|c|c|c|c|c|}
\hline & \multicolumn{2}{|c|}{ Total Sum of Donation } & \multicolumn{2}{|c|}{ Log $(1+$ Total Sum of Donation $)$} & \multicolumn{2}{|c|}{ Whether or Not Donation Was Given } \\
\hline & Experiment & Post-Experiment & Experiment & Post-Experiment & Experiment & Post-Experiment \\
\hline & $(1)$ & $(2)$ & $(3)$ & $(4)$ & $(5)$ & $(6)$ \\
\hline \multicolumn{7}{|l|}{ Panel A: Match Ratios and Urgency } \\
\hline $1: 1$ match & $\begin{array}{l}1.789 \\
(3.339)\end{array}$ & $\begin{array}{l}6.040^{* * *} \\
(2.314)\end{array}$ & $\begin{array}{l}0.008 \\
(0.037)\end{array}$ & $\begin{array}{l}0.055^{*} \\
(0.033)\end{array}$ & $\begin{array}{l}-0.002 \\
(0.009)\end{array}$ & $\begin{array}{l}0.013 \\
(0.008)\end{array}$ \\
\hline $1: 3$ match & $\begin{array}{l}0.327 \\
(3.160)\end{array}$ & $\begin{array}{l}6.256^{* * *} \\
(2.190)\end{array}$ & $\begin{array}{l}-0.027 \\
(0.035)\end{array}$ & $\begin{array}{l}0.028 \\
(0.031)\end{array}$ & $\begin{array}{l}-0.006 \\
(0.009)\end{array}$ & $\begin{array}{l}0.004 \\
(0.008)\end{array}$ \\
\hline September Mailer: Short Deadline & $\begin{array}{l}2.584 \\
(2.804)\end{array}$ & $\begin{array}{l}-5.434^{* * *} \\
(1.943)\end{array}$ & $\begin{array}{l}0.037 \\
(0.031)\end{array}$ & $\begin{array}{l}-0.048^{*} \\
(0.027)\end{array}$ & $\begin{array}{l}0.008 \\
(0.008)\end{array}$ & $\begin{array}{l}-0.010 \\
(0.007)\end{array}$ \\
\hline Mailers Contained Urgency Statement & $\begin{array}{l}0.591 \\
(2.097)\end{array}$ & $\begin{array}{l}-0.192 \\
(1.454)\end{array}$ & $\begin{array}{l}0.003 \\
(0.023)\end{array}$ & $\begin{array}{l}-0.001 \\
(0.020)\end{array}$ & $\begin{array}{l}0.001 \\
(0.006)\end{array}$ & $\begin{array}{l}0.000 \\
(0.005)\end{array}$ \\
\hline $\begin{array}{l}\text { September Mailer: Match Offer Framed In } \\
\text { Larger Terms (25:25 or } 25: 75)\end{array}$ & $\begin{array}{l}-2.661 \\
(2.653)\end{array}$ & $\begin{array}{l}-4.209^{* *} \\
(1.839)\end{array}$ & $\begin{array}{l}-0.078^{* * *} \\
(0.029)\end{array}$ & $\begin{array}{l}-0.046^{*} \\
(0.026)\end{array}$ & $\begin{array}{l}-0.019^{* * *} \\
(0.007)\end{array}$ & $\begin{array}{l}-0.011^{*} \\
(0.007)\end{array}$ \\
\hline Mean of Dependent Variable (Control) & 29.407 & 13.567 & 1.087 & 0.723 & 0.269 & 0.190 \\
\hline r2 & 0.221 & 0.150 & 0.193 & 0.095 & 0.149 & 0.088 \\
\hline $\mathrm{N}$ & 19959 & 19959 & 19959 & 19959 & 19959 & 19959 \\
\hline \multicolumn{7}{|l|}{ Panel B: Presence of Match and Urgency } \\
\hline Match & $\begin{array}{l}0.968 \\
(2.857)\end{array}$ & $\begin{array}{l}6.161^{* * *} \\
(1.980)\end{array}$ & $\begin{array}{l}-0.012 \\
(0.031)\end{array}$ & $\begin{array}{l}0.040 \\
(0.028)\end{array}$ & $\begin{array}{l}-0.004 \\
(0.008)\end{array}$ & $\begin{array}{l}0.008 \\
(0.007)\end{array}$ \\
\hline September Mailer: Short Deadline & $\begin{array}{l}2.695 \\
(2.794)\end{array}$ & $\begin{array}{l}-5.451^{* * *} \\
(1.937)\end{array}$ & $\begin{array}{l}0.039 \\
(0.031)\end{array}$ & $\begin{array}{l}-0.046^{*} \\
(0.027)\end{array}$ & $\begin{array}{l}0.008 \\
(0.008)\end{array}$ & $\begin{array}{l}-0.010 \\
(0.007)\end{array}$ \\
\hline Mailers Contained Urgency Statement & $\begin{array}{l}0.622 \\
(2.096)\end{array}$ & $\begin{array}{l}-0.196 \\
(1.453)\end{array}$ & $\begin{array}{l}0.004 \\
(0.023)\end{array}$ & $\begin{array}{l}-0.000 \\
(0.020)\end{array}$ & $\begin{array}{l}0.001 \\
(0.006)\end{array}$ & $\begin{array}{l}0.000 \\
(0.005)\end{array}$ \\
\hline $\begin{array}{l}\text { September Mailer: Match Offer Framed In } \\
\text { Larger Terms ( } 25: 25 \text { or } 25: 75)\end{array}$ & $\begin{array}{l}-2.654 \\
(2.653)\end{array}$ & $\begin{array}{l}-4.210^{* *} \\
(1.839)\end{array}$ & $\begin{array}{l}-0.078^{* * *} \\
(0.029)\end{array}$ & $\begin{array}{l}-0.045^{*} \\
(0.026)\end{array}$ & $\begin{array}{l}-0.019^{* * *} \\
(0.007)\end{array}$ & $\begin{array}{l}-0.011^{*} \\
(0.007)\end{array}$ \\
\hline Mean of Dependent Variable (Control) & 29.407 & 13.567 & 1.087 & 0.723 & 0.269 & 0.190 \\
\hline r2 & 0.221 & 0.150 & 0.193 & 0.095 & 0.149 & 0.088 \\
\hline $\mathrm{N}$ & 19959 & 19959 & 19959 & 19959 & 19959 & 19959 \\
\hline \multicolumn{7}{|l|}{ Panel C: Presence of Match, Urgency, and Interaction } \\
\hline Match & $\begin{array}{l}-4.101 \\
(3.602)\end{array}$ & $\begin{array}{l}4.802^{*} \\
(2.497)\end{array}$ & $\begin{array}{l}-0.002 \\
(0.040)\end{array}$ & $\begin{array}{l}0.032 \\
(0.035)\end{array}$ & $\begin{array}{l}0.001 \\
(0.010)\end{array}$ & $\begin{array}{l}0.005 \\
(0.009)\end{array}$ \\
\hline Interaction: Match*Urgency & $\begin{array}{l}10.247^{* *} \\
(4.436)\end{array}$ & $\begin{array}{l}2.747 \\
(3.075)\end{array}$ & $\begin{array}{l}-0.019 \\
(0.049)\end{array}$ & $\begin{array}{l}0.016 \\
(0.043)\end{array}$ & $\begin{array}{l}-0.011 \\
(0.012)\end{array}$ & $\begin{array}{l}0.006 \\
(0.011)\end{array}$ \\
\hline Mailers Contained Urgency Statement & $\begin{array}{l}-6.132^{*} \\
(3.598)\end{array}$ & $\begin{array}{l}-2.007 \\
(2.494)\end{array}$ & $\begin{array}{l}0.016 \\
(0.040)\end{array}$ & $\begin{array}{l}-0.011 \\
(0.035)\end{array}$ & $\begin{array}{l}0.008 \\
(0.010)\end{array}$ & $\begin{array}{l}-0.003 \\
(0.009)\end{array}$ \\
\hline September Mailer: Short Deadline & $\begin{array}{l}2.682 \\
(2.794)\end{array}$ & $\begin{array}{l}-5.454^{* * *} \\
(1.937)\end{array}$ & $\begin{array}{l}0.039 \\
(0.031)\end{array}$ & $\begin{array}{l}-0.046^{*} \\
(0.027)\end{array}$ & $\begin{array}{l}0.008 \\
(0.008)\end{array}$ & $\begin{array}{l}-0.010 \\
(0.007)\end{array}$ \\
\hline $\begin{array}{l}\text { September Mailer: Match Offer Framed In } \\
\text { Larger Terms ( } 25: 25 \text { or } 25: 75)\end{array}$ & $\begin{array}{l}-2.771 \\
(2.653)\end{array}$ & $\begin{array}{l}-4.242^{* *} \\
(1.839)\end{array}$ & $\begin{array}{l}-0.078^{* * *} \\
(0.029)\end{array}$ & $\begin{array}{l}-0.046^{*} \\
(0.026)\end{array}$ & $\begin{array}{l}-0.019^{* * *} \\
(0.007)\end{array}$ & $\begin{array}{l}-0.011^{*} \\
(0.007)\end{array}$ \\
\hline Mean of Dependent Variable (Control) & 29.407 & 13.567 & 1.087 & 0.723 & 0.269 & 0.190 \\
\hline r2 & 0.221 & 0.150 & 0.193 & 0.095 & 0.149 & 0.088 \\
\hline $\mathrm{N}$ & 19959 & 19959 & 19959 & 19959 & 19959 & 19959 \\
\hline \multicolumn{7}{|l|}{ Panel D: Urgency and Interaction of Framing and Ratios } \\
\hline $\begin{array}{l}\text { Interaction: } 1: 1 \text { Match * September Mailer: } \\
\quad \text { Match Offer Framed In Larger Terms }(25: 25)\end{array}$ & $\begin{array}{l}-1.452 \\
(3.241)\end{array}$ & $\begin{array}{l}-2.337 \\
(2.247)\end{array}$ & $\begin{array}{l}-0.009 \\
(0.036)\end{array}$ & $\begin{array}{l}-0.027 \\
(0.032)\end{array}$ & $\begin{array}{l}-0.003 \\
(0.009)\end{array}$ & $\begin{array}{l}-0.004 \\
(0.008)\end{array}$ \\
\hline $\begin{array}{l}\text { Interaction: } 1: 3 \text { Match * September Mailer: } \\
\text { Match Offer Framed In Larger Terms }(25: 75)\end{array}$ & $\begin{array}{l}-3.026 \\
(3.133)\end{array}$ & $\begin{array}{l}-1.246 \\
(2.172)\end{array}$ & $\begin{array}{l}-0.150^{* * *} \\
(0.035)\end{array}$ & $\begin{array}{l}-0.032 \\
(0.031)\end{array}$ & $\begin{array}{l}-0.036^{* * *} \\
(0.008)\end{array}$ & $\begin{array}{l}-0.012 \\
(0.008)\end{array}$ \\
\hline September Mailer: Short Deadline & $\begin{array}{l}3.096 \\
(2.504)\end{array}$ & $\begin{array}{l}-2.761 \\
(1.736)\end{array}$ & $\begin{array}{l}0.032 \\
(0.028)\end{array}$ & $\begin{array}{l}-0.029 \\
(0.024)\end{array}$ & $\begin{array}{l}0.006 \\
(0.007)\end{array}$ & $\begin{array}{l}-0.006 \\
(0.006)\end{array}$ \\
\hline Mailers Contained Urgency Statement & $\begin{array}{l}0.607 \\
(2.096)\end{array}$ & $\begin{array}{l}-0.255 \\
(1.453)\end{array}$ & $\begin{array}{l}0.003 \\
(0.023)\end{array}$ & $\begin{array}{l}-0.001 \\
(0.020)\end{array}$ & $\begin{array}{l}0.001 \\
(0.006)\end{array}$ & $\begin{array}{l}0.000 \\
(0.005)\end{array}$ \\
\hline Mean of Dependent Variable (Control) & 29.407 & 13.567 & 1.087 & 0.723 & 0.269 & 0.190 \\
\hline $\mathrm{r} 2$ & 0.221 & 0.149 & 0.194 & 0.095 & 0.149 & 0.088 \\
\hline $\mathrm{N}$ & 19959 & 19959 & 19959 & 19959 & 19959 & 19959 \\
\hline
\end{tabular}

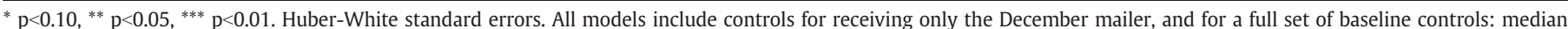

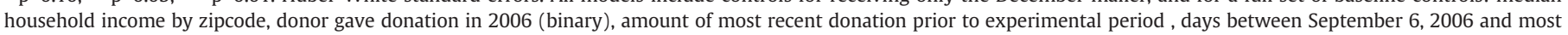

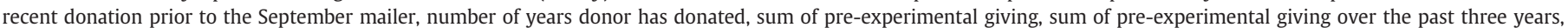

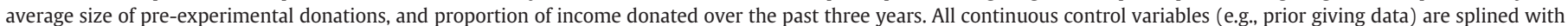
break points at each decile.

in the period ${ }^{5} ; M 1_{i}$ is equal to one if individual $i$ received a $\$ 1: \$ 1$ match offer; $M 3_{i}$ is equal to one if individual $i$ received a $\$ 1: \$ 3$ match offer; $M_{i}$ is equal to one if individual $i$ received any match offer; $S_{i}$ equals one if individual $i$ received a short deadline in her September mailer (September 31, 2006); $U_{i}$ equals one if individual $i$ received a

\footnotetext{
${ }^{5}$ When $Y_{\mathrm{i}}$ is binary, we also use probit specifications and find no differences in results. To avoid unnecessary functional form assumptions we report here the OLS specifications (Angrist, 2001).
}

mailer containing an urgency statement; $R_{i}$ equals one if individual $i$ received a match offer framed in larger terms $(25: 75,25: 25$ rather than $1: 1,1: 3)$; and $D_{i}$ equals one if individual $i$ received only the December mailer.

As Table 2 demonstrates, in the aggregate data the match offers had no statistically significant effect on any of the outcome measures within the experimental period. Similarly, the urgency treatment was insignificant in all specifications, except for a negative effect on amount donated in Panel C, and a positive interaction term of urgency 
and matching. Note the positive estimate on the interaction effect of the two treatments almost exactly counters the negative estimate of the two direct effects, thus in net the combined treatment of match and urgency is zero (whereas either treatment is actually negative, although only the urgency message is significant statistically). Thus, stating "now is the time to give" without giving a reason did harm, but then perhaps including the match provided a satisfactory reason and thus negated the harm (but did not actually lead to more giving, for the full sample frame).

Interestingly, we do find that the match offer has a significant effect on the amount donated in the post-experiment period (\$6.16). Yet, we do not consider this result strong since it loses statistical significance in the logarithmic specification (Column 3 ). This result is counter to Meier (2007), which found an increased giving during the matching period countered by a reduction after the matching offer ended. Since the experiment coincided with the end of the tax year and several major holidays, caution should be taken in generalizing this result without further experimentation. If there is seasonality in the price elasticity of demand for charitable giving, then the time frame may be of significance for this finding.

In almost all specifications, we also find that providing larger example amounts for the match (i.e., $\$ 25$ or $\$ 75$ rather than $\$ 1$ or $\$ 3$ ) has a negative effect on outcome measures, although this effect is only present for the smaller, 1:3 match ratio (Table 2 Panel D). This effect may be due to a misunderstanding of the offer, or negative affect if this was too high an amount (it is the average amount previously donated). Or, perhaps donors interpreted the $\$ 25: \$ 75$ frame as implying that the match required a $\$ 75$ donation or higher, thereby discouraging donors intending to donate less than $\$ 75$. (Alternatively, this misunderstanding could have also encouraged some donors to increase their donations to meet the perceived threshold.)

While we do not find evidence for a positive match effect in the experiment period, there is some important heterogeneity in treatment effects. Tables 3 and 4 present heterogeneous treatment effects (Table 3 shows the combined match treatment effect, and Table 4 separately estimates the two matching ratio treatment effects). Among donors who gave their most recent donation in the same calendar year (2006), we find that the match offer has a positive and significant effect on amount donated (\$12.91) relative to those who gave prior to 2006. Table 4 shows that the differential effect is larger ( $\$ 16.57$ vs. $\$ 9.22$ ) and more significant statistically for the smaller match offer (\$1:\$3), although statistical tests (not shown) comparing the point estimates for the two matches cannot rule out that they are homogeneous (and they are offset by the direct effect). Within this same sub-group, we also find that the match causes a 3.2\% increase in response rate relative to those who did not yet give in 2006 (who were 1.8 percentage points less likely to give in response to the match). This result is almost entirely driven by the $\$ 1: \$ 3$ match offer, which causes a $5.6 \%$ increase in response rate relative to those who did not yet give in 2006 (who were 3.1 percentage points less likely to give in response to the $\$ 1: \$ 3$ match). Appendices IV-VI demonstrate, through sample splits, that this effect only holds for donors who gave in the most recent calendar year, rather than a broader definition of "warm list" donors that includes all those who gave in the last 2 or 3 years.

\section{Conclusions}

This study uses a natural field experiment to further our insights on the economics of charity. In doing so, we are able to provide parameters of import to modelers interested in the underlying determinants of why and when people give, and supply practitioners with advice on how best to design their fundraising efforts.

Overall, the results confirm that a larger match offer ratio does not necessarily lead to larger, or more, charitable contributions, but that for certain populations and certain presentations the ratio may matter. If the maximum amount of a matching grant matters to donors, then in fact, it may be in the best interests of an organization to offer a smaller match offer ratio, given the opportunity cost associated with a higher match ratio offer. However, note that in Karlan and List (2007) the maximum amount was tested, and found to not generate any difference in likelihood of giving or amount given. Thus this would suggest a $\$ 1: \$ 1$ ratio is no more costly than a $\$ 1: \$ 3$ from the fundraisers perspective, and since the $\$ 1: \$ 3$ did do harm for some subsamples and presentations, the policy prescription from these two experiments put together suggests that a ratio at or above $\$ 1: \$ 1$ might be optimal.

Clearly, in addition to the empirical principles guiding this behavioral economic research, several features that characterize a particular context and donor heterogeneity can prove important to

Table 3

Heterogenous Treatment Effects, Total Sum of Donation \& Whether or Not Donation Was Given, Experiment Period.

\begin{tabular}{|c|c|c|c|c|c|c|}
\hline & \multicolumn{2}{|c|}{ Total amount of donation } & \multicolumn{2}{|c|}{$\log (1+$ Total amount of donation $)$} & \multicolumn{2}{|c|}{ Whether or not donation was given } \\
\hline & Experiment & Post-Experiment & Experiment & Post-Experiment & Experiment & Post-Experiment \\
\hline & $(1)$ & $(2)$ & (3) & $(4)$ & $(5)$ & $(6)$ \\
\hline \multicolumn{7}{|l|}{ Panel A: Match Ratios and Urgency } \\
\hline Match & $\begin{array}{l}-4.816 \\
(3.486)\end{array}$ & $\begin{array}{l}7.583^{* * *} \\
(2.417)\end{array}$ & $\begin{array}{l}-0.082^{* *} \\
(0.038)\end{array}$ & $\begin{array}{l}0.090^{* * *} \\
(0.034)\end{array}$ & $\begin{array}{l}-0.018^{*} \\
(0.009)\end{array}$ & $\begin{array}{l}0.021^{* *} \\
(0.009)\end{array}$ \\
\hline $\begin{array}{l}\text { Interaction: Match * Donated in year } \\
\text { prior to the experiment }\end{array}$ & $\begin{array}{l}12.910^{* * *} \\
(4.461)\end{array}$ & $\begin{array}{l}-3.173 \\
(3.092)\end{array}$ & $\begin{array}{l}0.157^{* * *} \\
(0.049)\end{array}$ & $\begin{array}{l}-0.113^{* * *} \\
(0.043)\end{array}$ & $\begin{array}{l}0.032^{* * *} \\
(0.012)\end{array}$ & $\begin{array}{l}-0.028^{* *} \\
(0.011)\end{array}$ \\
\hline Donated in year prior to the experiment & $\begin{array}{l}-27.046^{* * *} \\
(9.094)\end{array}$ & $\begin{array}{l}3.440 \\
(6.304)\end{array}$ & $\begin{array}{l}-0.333^{* * *} \\
(0.100)\end{array}$ & $\begin{array}{l}0.272^{* * * *} \\
(0.089)\end{array}$ & $\begin{array}{l}-0.069^{* * *} \\
(0.025)\end{array}$ & $\begin{array}{l}0.067^{* * * *} \\
(0.023)\end{array}$ \\
\hline September Mailer: Short Deadline & $\begin{array}{l}2.560 \\
(2.794)\end{array}$ & $\begin{array}{l}-5.418^{* * *} \\
(1.937)\end{array}$ & $\begin{array}{l}0.038 \\
(0.031)\end{array}$ & $\begin{array}{l}-0.045^{*} \\
(0.027)\end{array}$ & $\begin{array}{l}0.008 \\
(0.008)\end{array}$ & $\begin{array}{l}-0.009 \\
(0.007)\end{array}$ \\
\hline Mailers Contained Urgency Statement & $\begin{array}{l}0.577 \\
(2.096)\end{array}$ & $\begin{array}{l}-0.185 \\
(1.453)\end{array}$ & $\begin{array}{l}0.003 \\
(0.023)\end{array}$ & $\begin{array}{l}-0.000 \\
(0.020)\end{array}$ & $\begin{array}{l}0.001 \\
(0.006)\end{array}$ & $\begin{array}{l}0.001 \\
(0.005)\end{array}$ \\
\hline $\begin{array}{l}\text { September Mailer: Match Offer Framed In } \\
\text { Larger Terms }(25: 25 \mid 25: 75)\end{array}$ & $\begin{array}{l}-2.359 \\
(2.655)\end{array}$ & $\begin{array}{l}-4.283^{* *} \\
(1.840)\end{array}$ & $\begin{array}{l}-0.074^{* *} \\
(0.029)\end{array}$ & $\begin{array}{l}-0.048^{*} \\
(0.026)\end{array}$ & $\begin{array}{l}-0.018^{* *} \\
(0.007)\end{array}$ & $\begin{array}{c}-0.012^{*} \\
(0.007)\end{array}$ \\
\hline Mean of Dependent Variable (Control) & 29.407 & 13.567 & 1.087 & 0.723 & 0.269 & 0.190 \\
\hline r2 & 0.221 & 0.150 & 0.194 & 0.095 & 0.149 & 0.088 \\
\hline $\mathrm{N}$ & 19959 & 19959 & 19959 & 19959 & 19959 & 19959 \\
\hline
\end{tabular}

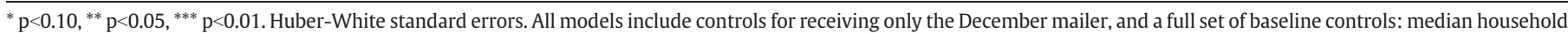

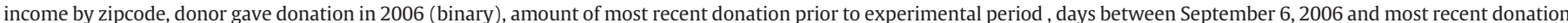

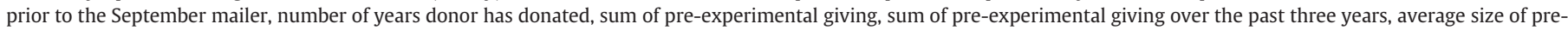

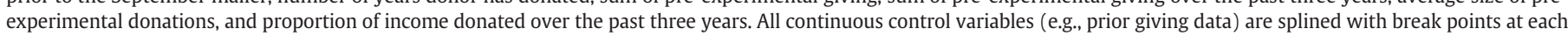
decile. 
Heterogenous Treatment Effects, Total Sum of Donation \& Whether or Not Donation Was Given.

\begin{tabular}{|c|c|c|c|c|c|c|}
\hline & \multicolumn{2}{|c|}{ Total amount of donation } & \multicolumn{2}{|c|}{$\log (1+$ Total amount of donation $)$} & \multicolumn{2}{|c|}{ Whether or not donation was given } \\
\hline & Experiment & Post-Experiment & Experiment & Post-Experiment & Experiment & Post-Experiment \\
\hline & $(1)$ & $(2)$ & (3) & $(4)$ & $(5)$ & $(6)$ \\
\hline \multicolumn{7}{|l|}{ Panel A: Match Ratios and Urgency } \\
\hline $1: 1$ match & $\begin{array}{l}-2.337 \\
(4.004)\end{array}$ & $\begin{array}{l}7.204^{* * *} \\
(2.776)\end{array}$ & $\begin{array}{l}-0.026 \\
(0.044)\end{array}$ & $\begin{array}{l}0.122^{* * *} \\
(0.039)\end{array}$ & $\begin{array}{l}-0.006 \\
(0.011)\end{array}$ & $\begin{array}{l}0.029 * * * \\
(0.010)\end{array}$ \\
\hline $1: 3$ match & $\begin{array}{l}-7.312^{*} \\
(3.956)\end{array}$ & $\begin{array}{l}7.961^{* * *} \\
(2.742)\end{array}$ & $\begin{array}{l}-0.139^{* * *} \\
(0.044)\end{array}$ & $\begin{array}{l}0.059 \\
(0.039)\end{array}$ & $\begin{array}{l}-0.031^{* * *} \\
(0.011)\end{array}$ & $\begin{array}{l}0.013 \\
(0.010)\end{array}$ \\
\hline $\begin{array}{l}\text { Interaction: } 1: 1 \text { match } * \text { Donated in year } \\
\text { prior to the experiment }\end{array}$ & $\begin{array}{l}9.218^{*} \\
(5.269)\end{array}$ & $\begin{array}{l}-2.653 \\
(3.653)\end{array}$ & $\begin{array}{l}0.070 \\
(0.058)\end{array}$ & $\begin{array}{l}-0.159^{* * *} \\
(0.051)\end{array}$ & $\begin{array}{l}0.008 \\
(0.014)\end{array}$ & $\begin{array}{l}-0.038^{* * *} \\
(0.013)\end{array}$ \\
\hline $\begin{array}{l}\text { Interaction: } 1: 3 \text { match }{ }^{*} \text { Donated in year } \\
\text { prior to the experiment }\end{array}$ & $\begin{array}{l}16.565^{* * *} \\
(5.163)\end{array}$ & $\begin{array}{l}-3.695 \\
(3.579)\end{array}$ & $\begin{array}{l}0.242^{* * *} \\
(0.057)\end{array}$ & $\begin{array}{l}-0.067 \\
(0.050)\end{array}$ & $\begin{array}{l}0.054^{* * *} \\
(0.014)\end{array}$ & $\begin{array}{l}-0.019 \\
(0.013)\end{array}$ \\
\hline Donated in year prior to the experiment & $\begin{array}{l}-27.113^{* * *} \\
(9.096)\end{array}$ & $\begin{array}{l}3.452 \\
(6.305)\end{array}$ & $\begin{array}{l}-0.335^{* * *} \\
(0.100)\end{array}$ & $\begin{array}{l}0.271^{* * *} \\
(0.089)\end{array}$ & $\begin{array}{l}-0.069^{* * *} \\
(0.025)\end{array}$ & $\begin{array}{l}0.067^{* * *} \\
(0.023)\end{array}$ \\
\hline September Mailer: Short Deadline & $\begin{array}{l}2.280 \\
(2.806)\end{array}$ & $\begin{array}{l}-5.374^{* * *} \\
(1.945)\end{array}$ & $\begin{array}{l}0.031 \\
(0.031)\end{array}$ & $\begin{array}{l}-0.048^{*} \\
(0.027)\end{array}$ & $\begin{array}{l}0.007 \\
(0.008)\end{array}$ & $\begin{array}{l}-0.010 \\
(0.007)\end{array}$ \\
\hline Mailers Contained Urgency Statement & $\begin{array}{l}0.570 \\
(2.097)\end{array}$ & $\begin{array}{l}-0.183 \\
(1.454)\end{array}$ & $\begin{array}{l}0.003 \\
(0.023)\end{array}$ & $\begin{array}{l}-0.000 \\
(0.020)\end{array}$ & $\begin{array}{l}0.001 \\
(0.006)\end{array}$ & $\begin{array}{l}0.000 \\
(0.005)\end{array}$ \\
\hline $\begin{array}{l}\text { September Mailer: Match Offer Framed In } \\
\quad \text { Larger Terms }(25: 25 \mid 25: 75)\end{array}$ & $\begin{array}{l}-2.152 \\
(2.659)\end{array}$ & $\begin{array}{l}-4.312^{* *} \\
(1.844)\end{array}$ & $\begin{array}{l}-0.069 * * \\
(0.029)\end{array}$ & $\begin{array}{l}-0.045^{*} \\
(0.026)\end{array}$ & $\begin{array}{l}-0.017^{* *} \\
(0.007)\end{array}$ & $\begin{array}{l}-0.012^{*} \\
(0.007)\end{array}$ \\
\hline Mean of Dependent Variable (Control) & 29.407 & 13.567 & 1.087 & 0.723 & 0.269 & 0.190 \\
\hline r2 & 0.221 & 0.150 & 0.194 & 0.095 & 0.149 & 0.088 \\
\hline $\mathrm{N}$ & 19959 & 19959 & 19959 & 19959 & 19959 & 19959 \\
\hline
\end{tabular}

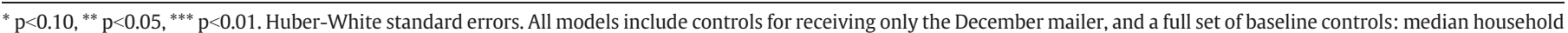

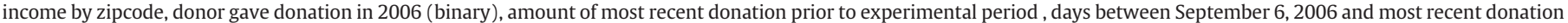

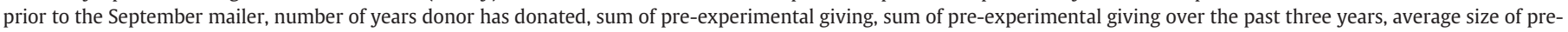

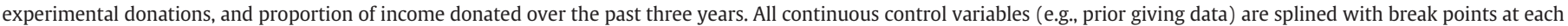
decile.

understanding how to optimize fundraising outcomes. For example, those who have given less recently respond negatively to the match, and even more negatively to the $\$ 1: \$ 3$ matching ratio, but those who have given more recently prior to the matching offer do not have negative responses to the match.

On the one hand, that pattern is generally consistent with other findings that show relative "scope insensitivity" (Frederick and Fischhoff, 1998); at the same time, there might be instances, depending on the type of donors, their expertise and dedication, where match ratios might have a greater impact. Along similar lines, one can imagine scenarios (e.g., unique dramatic events, such as natural disasters) where a message of urgency might prove more compelling than it did in the present case, or scenarios where the offer of a match might garner greater attention. Interestingly, in the present study, while each of these - urgency and match - proved rather ineffectual during the fundraising period, their co-occurrence seemed to have been mutually reinforcing, as if convincing potential donors that the cause might indeed be in need of greater timely support.

On a more practical level, these findings suggest that the loyalty or attention of a donor not only affects the likelihood of giving and amount given, but also affects the relative performance of different fundraising approaches. Although customization of donor appeals is costly, this suggests that there are rewards to understanding the mindset of the donor in order to send the optimal appeal. Whether these rewards outweigh the costs is of course an empirical question.

A clear feature of fundraising that is likely to impact its success is the attention it is able to garner among potential donors. While not all those who are aware of a fundraising drive or special offer will contribute, lack of awareness clearly is likely to reduce giving. In the present study, we observed significant differences between those who had given to the organization in the past year as compared to those who had not. Given the rate at which this organization sends its solicitations, those who had not contributed for a year or more will have typically foregone responding to at least four mailers. It is possible that among these recipients attention to the mailer was lower - in terms of opening the mailer, reading it, or retaining it for further consideration - than among those who contributed, thus showing interest, during a more recent period.

Of course, potential donors' attention to mail solicitations is a familiar concern in fundraising. Whereas some manipulations, such as colors and photos on the envelope, might alter the likelihood that a recipient would inspect her mail, other features, such as varying amounts of a match offer, are only likely to play a role once attention is already given. Similar concerns regarding influence through central versus peripheral routes are discussed in Bertrand et al. (2010). Further research along the lines presented here can help elucidate the relationship between kinds of solicitation offers and type of potential donors in the hopes of making costly fundraising optimally effective.

\section{Appendix A. Supplementary data}

Supplementary data to this article can be found online at doi:10.1016/j.jpubeco.2010.11.024.

\section{References}

Andreoni, James, 2006. Philanthropy. In: Kolm, Serge C., Ythier, Jean M. (Eds.), Handbook of Giving, Reciprocity, and Altruism and Reciprocity. Elsevier, pp. 1201-1269.

Andreoni, James, 2007. Charitable giving, The New Palgrave Dictionary of Economics 2nd Edition.

Andreoni, James and Payne, A. Abigail, forthcoming. Is Crowding Out Due Entirely to Fundraising? Evidence from a Panel of Charities. Journal of Public Economics.

Angrist, Joshua, 2001. Estimation of limited dependent variable models with dummy endogenous regressors: simple strategies for empirical practice. Journal of Business \& Economic Statistics 19 (1), 2-16.

Auten, Gerald, Sieg, Holger, Clotfelter, Charles T., 2002. Charitable giving, income and taxes: an analysis of panel data. American Economic Review 92 (1), 371-382.

Bertrand, M., Karlan, D., Mullainathan, S., Shafir, E., Zinman, J., 2010. What's Advertising Content Worth? Evidence from a Consumer Credit Marketing Field Experiment. The Quarterly Journal of Economics 125 (1), 263-305.

Clotfelter, Charles T., 1985. Federal tax policy and charitable giving. University of Chicago Press, Chicago.

Croson, Rachel, Shang, Jen, 2005. Field experiments in charitable contribution: the impact of social influence on the voluntary provision of public goods. Working paper, Wharton.

Croson, Rachel, Shang, Jen, 2008. The impact of downward social information on contribution decisions. Experimental Economics 11 (3), 221-233. 
Eckel, Catherine C., Grossman, Philip J., 2003. Rebate versus matching: does how we subsidize charitable contributions matter? Journal of Public Economics 87 (3-4), 681-701.

Feldstein, Martin, 1975. The income tax and charitable contributions: part II - the impact on religious, educational and other organizations. National Tax Journal 28 (2), 209-226.

Frederick, S., Fischhoff, B., 1998. Scope (in)sensitivity in elicited valuations. Risk Decisions and Policy 3 (2), 109-123.

Frey, Bruno S., Meier, Stephan, 2004. Social comparisons and pro-social behavior: testing conditional cooperation in a field experiment. American Economic Review 94 (5), 1717-1722.

Karlan, Dean, List, John A., 2007. Does price matter in charitable giving? evidence from a large-scale natural field experiment. American Economic Review 97 (5), 1774-1793.

Levitt, Steven D., List, John A., 2007. What do laboratory experiments measuring social preferences reveal about the real world? Journal of Economic Perspectives 21 (2), 153-174.
List, John A., forthcoming. The Market for Charitable Giving. Journal of Economic Perspectives.

List, John A., Lucking-Reiley, David, 2002. The effects of seed money and refunds on charitable giving: experimental evidence from a university capital campaign. Journal of Political Economy 110 (1), 215-233.

McManus, Brian and Bennet, Rick, forthcoming. The Demand for Products Linked to Public Goods: Evidence from an Online Field Experiment. Journal of Public Economics.

Meier, Stephan, 2007. Do subsidies increase charitable giving in the long-run? matching donations in a field experiment. Journal of the European Economic Association 5 (6), 1203-1222.

Randolph, William C., 1995. Dynamic income, progressive taxes, and the timing of charitable contributions. Journal of Political Economy 103 (4), 709-738.

Rondeau, Daniel, List, John A., 2008. Matching and challenge gifts to charity: evidence from laboratory and natural field experiments. Experimental Economics 11 (3), 253-267. 